Applied Mathematical Sciences, Vol. 9, 2015, no. 12, $597-602$
HIKARI Ltd, www.m-hikari.com
http://dx.doi.org/10.12988/ams.2015.410854

\title{
Tree Cover of the Join and the Corona of Graphs
}

\author{
Rosalio G. Artes, Jr., and Rene D. Dignos \\ Department of Mathematics and Statistics \\ College of Science and Mathematics, MSU - Iligan Institute of Technology \\ Andres Bonifacio Avenue, Tibanga, 9200 Iligan City, Philippines \\ Copyright (C) 2014 Rosalio G. Artes, Jr., and Rene D. Dignos. This is an open access \\ article distributed under the Creative Commons Attribution License, which permits unre- \\ stricted use, distribution, and reproduction in any medium, provided the original work is \\ properly cited.
}

\begin{abstract}
Let $G$ be a graph and $\mathscr{T}_{G}=\left\{G_{1}, G_{2}, G_{3}, \ldots, G_{k}\right\}$ be a collection of subgraphs of $G$ where $G_{i}$ is a subtree of $G$ for every $i \in\{1,2, \ldots, k\}$. If for every edge $e \in E(G)$, there exists $G_{i} \in \mathscr{T}_{G}$ such that $e \in E\left(G_{i}\right)$, then $\mathscr{T}_{G}$ is a tree cover of $G$. The tree covering number of $G$ is the minimum cardinality among the tree covers of $G$. In this paper, we establish some bounds for the tree covering numbers of the join and the corona of two vertex-disjoint graphs.
\end{abstract}

Mathematics Subject Classification: 05C05, 05C30

Keywords: tree cover, tree covering number, join, corona

\section{Introduction}

Let $G$ be a simple graph. A subtree of $G$ is a connected acyclic subgraph of $G$. A collection $\mathscr{T}_{G}=\left\{G_{1}, G_{2}, G_{3}, \ldots, G_{k}\right\}$ of subgraphs of $G$ is a tree cover of $G$ if $G_{i}$ is a subtree of $G$ for every $i \in\{1,2, \ldots, k\}$ and for every edge $e \in E(G)$, there exists $G_{i} \in \mathscr{T}_{G}$ such that $e \in E\left(G_{i}\right)$. The tree covering number of $G$, denoted by $t_{c}(G)$, is given by $t_{c}(G)=\min \left\{\left|\mathscr{T}_{G}\right|: \mathscr{T}_{G}\right.$ is a tree cover of $\left.G\right\}$.

The graph $G$ in Figure 1.1 has tree covering number equal to 2 . 


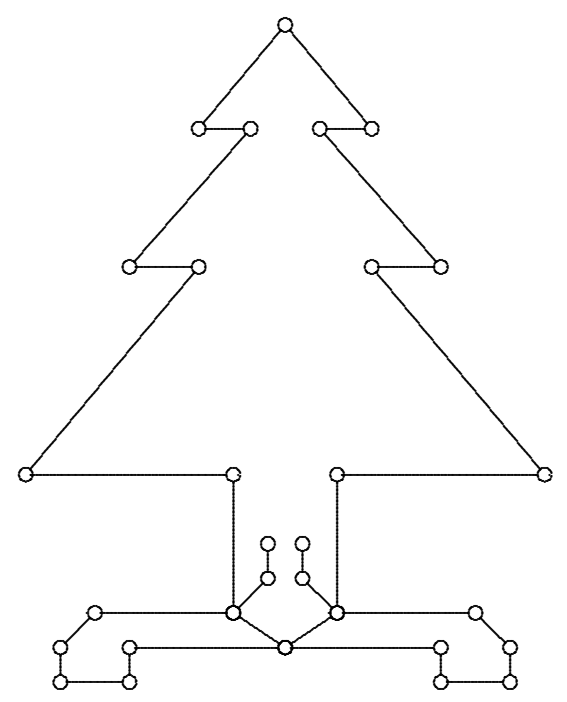

Figure 1.1: A graph $G$ with $t_{c}(G)=2$.

Indeed, let $\mathscr{T}_{G}=\left\{G_{1}, G_{2}\right\}$, where $G_{1}$ and $G_{2}$ are subgraphs of $G$ shown in Figure 1.2 and Figure 1.3, respectively.

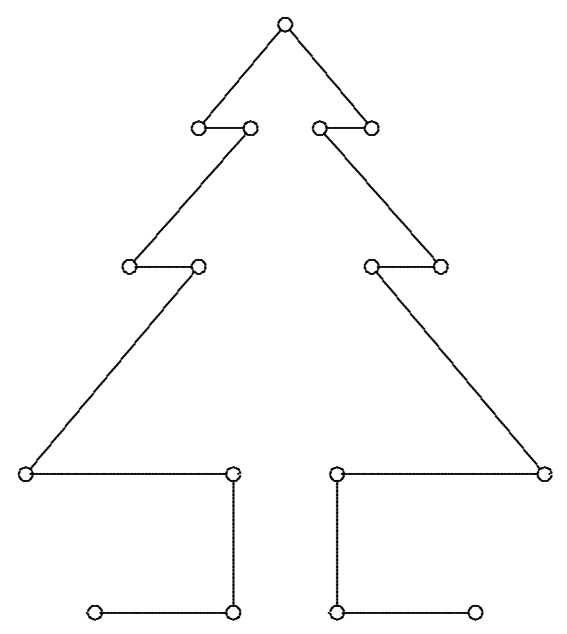

Figure 1.2: The graph $G_{1}$.

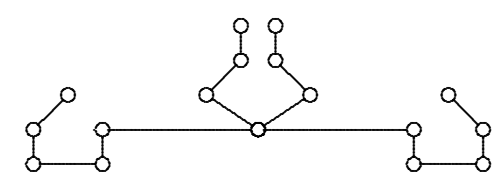

Figure 1.3: The graph $G_{2}$. 
Clearly, $G_{1}$ and $G_{2}$ are subtrees of $G$. Moreover, $G_{1} \cup G_{2}=G$. Hence, every edge of $G$ is either in $G_{1}$ or in $G_{2}$. Consequently, $\mathscr{T}_{G}$ is a tree cover of $G$. Thus, by definition, $t_{c}(G) \leq\left|\mathscr{T}_{G}\right|=2$. Since $G$ is not a tree, Theorem 2.2 found in [1] asserts that $t_{c}(G) \geq 2$. Combining the two inequalities gives $t_{c}(G)=2$.

The next section establishes an upper bound for the tree covering number of the join of two vertex-disjoint graphs.

\section{Tree Covering Number of the Join of Graphs}

Here, we formally define the join of two vertex-disjoint graphs.

Definition 2.1 [4] Let $G$ and $H$ be vertex-disjoint graphs. The join $G \oplus H$ of $G$ and $H$ has vertex-set $V(G \oplus H)=V(G) \cup V(H)$ and edge-set

$$
E(G \oplus H)=E(G) \cup E(H) \cup\{u v: u \in V(G), v \in V(H)\}
$$

Consequently,

$$
|V(G \oplus H)|=|V(G)|+|V(H)|
$$

and

$$
|E(G \oplus H)|=|E(G)|+|E(H)|+|V(G)||V(H)| .
$$

Note also that the operation $\oplus$ is commutative, i.e., $G \oplus H$ is isomorphic to $H \oplus G$ with respect to adjacency.

Let us consider an illustration of the above definition.

Example 2.2 Consider the path $P_{9}$ and the complete graph $K_{1}$ with vetexset $V\left(K_{1}\right)=\{u\}$. Then the join of $P_{9}$ and $K_{1}$ is shown below.

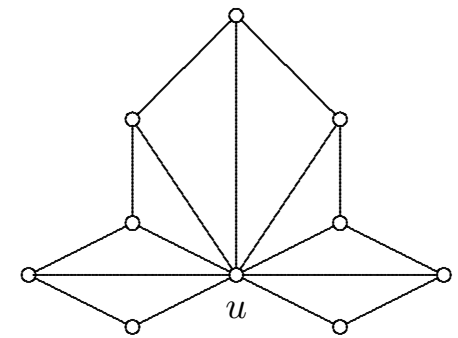

Figure 2.1: The join $P_{9}+K_{1}$.

In the above illustration, every vertex of $P_{9}$ is joined with the vertex $u$.

An upper bound for the tree covering number of the join of two vertexdisjoint graphs is established in the following theorem. 
Theorem 2.3 Let $G$ an $H$ be two vertex-disjoint graphs of orders $m$ and $n$, respectively. Then,

$$
t_{c}(G \oplus H) \leq \min \left\{m+t_{c}(H), n+t_{c}(G)\right\} .
$$

Proof: Let $V(G)=\left\{v_{1}, v_{2}, v_{3}, \ldots, v_{m}\right\}$ and $V(H)=\left\{u_{1}, u_{2}, u_{3}, \ldots, u_{n}\right\}$. Then $v_{i} u_{j} \in E(G \oplus H)$ for all $i=1,2,3, \ldots, m$ and for all $j=1,2,3, \ldots, n$. Let $\mathscr{T}_{G}$ and $\mathscr{T}_{H}$ be tree covers of $G$ and $H$, respectively, such that $\left|\mathscr{T}_{G}\right|=t_{c}(G)$ and $\left|\mathscr{T}_{H}\right|=t_{c}(H)$. Now, for every vertex $v \in V(G),\langle\{v\}\rangle \oplus \overline{H \cup \bar{H}}$ is a star, and hence a tree. Moreover, there exists $G_{v} \in \mathscr{T}_{G}$ with $v \in V(G)$ and $G_{v} \cup\langle\{v\}\rangle \oplus \overline{H \cup \bar{H}}$ is a tree. Let

$$
\mathscr{T}_{G+H}=\mathscr{T}_{H} \cup\left\{G_{v} \cup\langle\{v\}\rangle \oplus \overline{H \cup \bar{H}}: v \in V(G)\right\}
$$

Then $\mathscr{T}_{G \oplus H}$ is a tree cover of $G \oplus H$. Thus,

$$
t_{c}(G \oplus H) \leq\left|\mathscr{T}_{H}\right|+\left|\left\{G_{v} \cup\langle\{v\}\rangle \oplus \overline{H \cup \bar{H}}: v \in V(G)\right\}\right| .
$$

But $\left|\mathscr{T}_{H}\right|=t_{c}(H)$ and $\left|\left\{G_{v} \cup\langle\{v\}\rangle \oplus \overline{H \cup \bar{H}}: v \in V(G)\right\}\right|=|V(G)|=m$. Thus,

$$
t_{c}(G \oplus H) \leq m+t_{c}(H)
$$

Similarly,

$$
t_{c}(G \oplus H) \leq n+t_{c}(G)
$$

Combining Inequalities (1) and (2) gives the desired result.

Consider now the complete bipartite graph $K_{m, n}$. An upper bound for its tree covering number is established in the following theorem.

Theorem 2.4 Let $m$ and $n$ be positive integers. Then, $t_{c}\left(K_{m, n}\right) \leq \min \{m, n\}$.

Proof: Note that $K_{m, n}=\overline{K_{m}} \oplus \overline{K_{n}}$. Thus by Theorem 2.3,

$$
\begin{aligned}
t_{c}\left(K_{m, n}\right) & =t_{c}\left(\overline{K_{m}} \oplus \overline{K_{n}}\right) \\
& \leq \min \left\{m+t_{c}\left(\overline{K_{n}}\right), n+t_{c}\left(\overline{K_{m}}\right)\right\} \\
& =\min \{m+0, n+0\} \\
& =\min \{m, n\} .
\end{aligned}
$$

Thus, $t_{c}\left(K_{m, n}\right) \leq \min \{m, n\}$.

The tree covering number of the corona of graphs is established in the following section. 


\section{Tree Covering Number of the Corona of Graphs}

Formally, we define the corona of two vertex-disjoint graphs.

Definition 3.1 [4] The corona $G \circ H$ of two graphs $G$ and $H$ is the graph obtained by taking one copy of $G$ of order $n$ and $n$ copies of $H$, and then joining the $i^{t h}$ vertex of $G$ to every vertex in the $i^{t h}$ copy of $H$.

Example 3.2 The figure below illustrates the corona $P_{3} \circ C_{3}$.

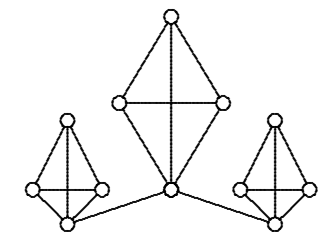

Figure 3.1: The corona $P_{3} \circ C_{3}$.

Note that if $H$ is a tree, then $G \circ H$ has tree covering number equal to the tree covering number of $G$. We formally write this result in the following theorem.

Theorem 3.3 Let $G$ be a connected graph. Then for any tree $T, t_{c}(G \circ T)=$ $t_{c}(G)$.

Next, we give an upper bound for the tree covering number of the corona of two graphs as a linear combination of the tree covering number of $G$ and the tree covering number of $H$.

Theorem 3.4 Let $G$ and $H$ be two nontrivial connected graphs of orders $m$ and $n$, respectively. Then $t_{c}(G \circ H) \leq t_{c}(G)+m t_{c}(H)$.

Proof: Let $\mathscr{T}_{G}$ and $\mathscr{T}_{H}$ be tree covers of $G$ and $H$, respectively, such that $\left|\mathscr{T}_{G}\right|=t_{c}(G)$ and $\left|\mathscr{T}_{H}\right|=t_{c}(H)$. For every $u \in V(G)$, each copy $H_{u}$ of $H$ can be covered by $\left|\mathscr{T}_{H}\right|$ subtrees. Hence, all the copies of $H$ in $G \circ H$ can be covered by $m t_{c}(H)$ subtrees. Now, for every vertex $v \in V(G)$, there exists $G_{v} \in \mathscr{T}_{G}$ with $v \in G_{v}$ and $G_{v} \cup\langle\{v\}\rangle \oplus \overline{H \cup \bar{H}}$ is a tree. Thus, the family $\mathscr{T}_{G \circ H}=\bigcup\left\{\mathscr{T}_{H_{u}}: u \in V(G)\right\} \cup\left\{G_{v} \cup\langle\{v\}\rangle \oplus \overline{H \cup \bar{H}}\right\}$ is a tree cover of $G \circ H$. Accordingly,

$$
\begin{aligned}
t_{c}(G \circ H) & \leq\left|\bigcup\left\{\mathscr{T}_{H_{u}}: u \in V(G)\right\} \cup\left\{G_{v} \cup\langle\{v\}\rangle \oplus \overline{H \cup \bar{H}}\right\}\right| \\
& =m\left|\mathscr{T}_{H}\right|+\left|\mathscr{T}_{G}\right| \\
& =m t_{c}(H)+t_{c}(G) .
\end{aligned}
$$

This completes the proof. 


\section{References}

[1] R.G. Artes, Jr. and R.D. Dignos, Tree Cover of Graphs, Applied Mathematical Sciences, 8 (201), 7469-7473.

http://dx.doi.org/10.12988/ams.2015.49728

[2] G. Chartrand and L. Lesniak, Graphs and Digraphs, New York: Chapman and Hall, 1996.

[3] G. Chartrand and O.R. Oellermann, Applied and Algorithmic Graph Theory, New York: McGraw-Hill, Inc., 1993.

[4] F. Harary, Graph Theory, Addison Wesley Publishing Co. Inc., 1972.

[5] K. Thulasiraman and M.N.S Swamy, Graph Theory and Algorithms, A Wiley-Interscience Publication, Canada, 1992. http://dx.doi.org/10.1002/9781118033104

Received: November 5, 2014; Published: January 12, 2015 